\title{
BMJ Open Systematic literature review of the burden and outcomes of infections due to multidrug-resistant organisms in Europe: the ABOUT-MDRO project protocol
}

\author{
Blanca Anaya-Baz, ${ }^{1}$ Natalia Maldonado, ${ }^{1}$ Zaira R Palacios-Baena (D) , ${ }^{1}$ \\ Virginia Palomo, ${ }^{1}$ Maria Diletta Pezzani, ${ }^{2}$ Sheila Chiesi (1) , ${ }^{2}$ Elisa Razzaboni, ${ }^{2}$ \\ Monica Compri, ${ }^{2}$ Evelina Tacconelli, ${ }^{2}$ Jesús Rodriguez-Baño ${ }^{1}$
}

To cite: Anaya-Baz B, Maldonado N, PalaciosBaena ZR, et al. Systematic literature review of the burden and outcomes of infections due to multidrug-resistant organisms in Europe: the ABOUT-MDRO project protocol. BMJ Open 2020;10:e030608. doi:10.1136/ bmjopen-2019-030608

- Prepublication history and additional material for this paper are available online. To view these files, please visit the journal online (http://dx.doi. org/10.1136/bmjopen-2019030608).

Received 25 March 2019 Revised 25 September 2019 Accepted 03 March 2020
Check for updates

(C) Author(s) (or their employer(s)) 2020. Re-use permitted under CC BY-NC. No commercial re-use. See rights and permissions. Published by BMJ.

For numbered affiliations see end of article.

Correspondence to Dr Jesús Rodriguez-Baño; jesusrb@us.es

\section{ABSTRACT}

Introduction Despite the increasing importance of infections due to multidrug-resistant organisms (MDROs), there is a lack of comprehensive information about the burden of disease and outcomes of key infections caused by these pathogens. The aim of the ABOUT-MDRO (A systematic review on the burden and outcomes of infections due to multidrug resitant organisms) project is to provide estimations of the burden of some key infections and their outcomes caused by the target MDROs.

Methods and analysis A systematic literature search will be performed using MEDLINE/PubMed, Elsevier's SCOPUS, Cochrane library, Clinical trials and Web of Science, as well as the Surveillance Systems from Public Health Institutions and Scientific Societies for Antimicrobial Resistance and Healthcare-Associated Infections in Europe database of European surveillance systems, for data on prevalence/ incidence, mortality and length of stay of target infections in hospitalised patients (including ventilator-associated pneumonia, hospital-acquired pneumonia, complicated intra-abdominal infections, complicated urinary tract infections, skin and soft tissue infections and bloodstream infections) and in specific populations (children, hospital wards, neutropenic patients) caused by cephalosporinresistant or carbapenem-resistant Enterobacteriaceae, carbapenem-resistant $P$ seudomonas aeruginosa and Acinetobacter spp., methicillin-resistant Staphylococcus aureus, and vancomycin-resistant Enterococcus spp. The information retrieved will be tabulated and pooled estimates and $95 \%$ Cls calculated of rates and outcomes, using random effects models. Relationships between rates and outcomes in randomised control trials and epidemiological studies, and data of proportions and incidence/prevalence rates will also be analysed. The information collected in this study will be useful for identifying gaps in our knowledge in terms of incidence/ prevalence and clinical outcomes of infections caused by MDROs, and for informing priorities in infection control and the research and design of appropriate studies.

Ethics and dissemination This study will be based on published data so we did not require ethical approval. Formal consent is not required. The results of this review
Strengths and limitations of this study.

- Data synthesis will involve collection and summary of available data on rates and outcome estimations of different types of infections caused by prioritised antibiotic-resistant pathogens, overall and for specific patient populations and geographical areas in Europe.

- Previous databases for infections and pathogens surveillance identified by systematic reviews from EPI-net (https://epi-net.eu/) such as Surveillance Systems from Public Health Institutions and Scientific Societies for Antimicrobial Resistance and Healthcare-Associated Infections in Europe (doi: 10.1016/j.cmi.2017.07.014) will be available.

- Data from sources of information not publicly available will be missed. Also, available data are expected to be scarce for some infections. However, the study will also be useful to identify the gaps in information.

- Definitions for outcomes and methodology for follow-up of patients may be heterogeneous across studies.

will be reported according to the Preferred Reporting Items for Systematic Reviewand Meta-Analyses statement. Data will be presented at international conferences and published in peer-reviewed journals.

Registration details PROSPERO (https://www.crd.york. ac.uk/prospero/) (CRD42019124185).

\section{INTRODUCTION}

The worldwide spread of organisms that are resistant to multiple antibiotics, generally referred to as multidrug-resistant organisms (MDRO), has become a public health concern. ${ }^{2}{ }^{2}$ The WHO recently established a priority list of microorganisms to inform research priorities for the development of new antibiotics, with carbapenem-resistant 
Acinetobacter baumannii (CRAB) and Pseudomonas aeruginosa, third-generation or carbapenem-resistant Enterobacteriaceae, vancomycin-resistant Enterococcus spp. and methicillin-resistant Staphylococcus aureus considered critical and of high priority. ${ }^{3}$ There is also an urgent need for improvements in surveillance to help optimise empirical therapy, drive antimicrobial stewardship and infection control measures, and help in the rational use of old and new drugs against resistant organisms. ${ }^{4}$

The main sources of information on rates of infection caused by MDROs come from regional, national or international surveillance systems, published epidemiological studies and outbreak reports. Two databases developed in the context of EPI-NET (epidemiologic European network)/Combatting Bacterial Resistance in Europe Molecules against Gram-negative Infections consortium identified relevant sources of information: the SUSPIRE Study (Surveillance Systems from Public Health Institutions and Scientific Societies for Antimicrobial Resistance and Healthcare-Associated Infections in Europe) recently mapped all surveillance systems with publicly available data in Europe and showed that, despite recent improvements and studies which control and study this issue, the available data still remain to be excessively heterogeneous and scarce to generate an exhaustive correlation and comparison with publicly available surveillance systems information across Europe. $^{5}$ Additionally, published outbreak reports were mapped by the EMBARGO (EpideMiology and control measures of outBreaks due to Antibiotic-Resistant orGanisms in EurOpe) Study. ${ }^{6}$ While frequent use is made of data from surveillance systems and studies providing proportions of isolates with resistance to specific antimicrobials, comprehensive data on pathogen incidence rates and infections are very limited. This is important because data based on proportion of resistant isolates can be misleading if not adequately interpreted; to give an example, if hospital A found two carbapenem-resistant A. baumannii out of 4 isolates of this pathogen in 1 year, while hospital $\mathrm{B}$, despite having a similar number of admissions, found 20/40, in both cases, the proportion is $50 \%$, but the burden of infection caused by carbapenem-resistant $A$. baumannii is very different. Furthermore, the clinical burden varies according to the predominant infection type or population affected. To have such data available would be of critical importance for informing the public, as well as policy makers, hospital managers, research funding bodies and companies working on the development of diagnostic and therapeutic tools for priority research and infection control activities. The clinical impact of MDRO infections is usually measured using crude mortality and length of overall hospital stay. The studies attempting to estimate the attributable influence of antimicrobial resistance on the outcome of patients are heterogeneous in the populations included and in the methodology. ${ }^{7-11}$

Overall, in view of the data provided, the main research question is: What is the burden of infections and resistance caused by MDROs overall and for specific types of infections, microorganisms and patients in the different countries/geographical areas/hospitals in Europe, and which is the clinical burden due to carbapenem-resistant Klebsiella pneumoniae, CRAB and vancomycin-resistant Enterococci (VRE)?

The major aim of the ABOUT-MDRO project (a systematic review on the burden and outcomes of infections due to MDRO) is to estimate the burden of key infections and their outcomes caused by target MDROs.

\section{METHODS/DESIGN \\ Design}

A systematic review of the scientific and grey literature will be performed. The target MDROs for this study are carbapenem-resistant Acinetobacter spp and P. aeruginosa; cephalosporin-resistant or carbapenem-resistant Enterobacteriaceae; vancomycin-resistant Enterococcus spp., and methicillin-resistant $S$. aureus. The target infections include ventilator-associated pneumonia and hospitalacquired pneumonia, complicated intra-abdominal infections, complicated urinary tract infections, complicated skin and skin structure infections, and bloodstream infections.

\section{Eligibility criteria}

Studies will be considered for inclusion if providing any of the following, for the target infections caused by the target MDROs occurring in hospitalised patients: incidence rate, incidence density, prevalence, all-cause mortality or length of stay. Studies not providing the estimations but for which the numerators and denominators for their calculation is available will also be included. All other eligibility criteria are shown in box 1 . Studies from systematic reviews will only be included if the data from the individual studies included in them are not available.

\section{Information sources}

This systematic review followed the Preferred Reporting Items for Systematic Reviewand Meta-Analysis Protocols guidelines scheme (online supplementary figure S1) and checklist (online supplementary table S1) for methodology development and structure research. The following electronic databases will be searched: MEDLINE-PubMed, Cochrane library, SCOPUS and Web of Science. The updated surveillance systems databases for antimicrobialresistant pathogens and hospital-acquired infections from the SUSPIRE project ${ }^{5}$ and EPI-NET (https://epinet.eu/) will also be searched.

\section{Search strategy}

A comprehensive search strategy will be performed for peer-reviewed literature, with no language restrictions. The search strategy will be performed using MeSH (Medical Subject Headings) terms and No-MeSH terms, selected after piloting different search strategies. Finally, and according to the piloting, No-MeSH search terms 


\section{Box 1 Inclusion criteria}

- Study designs: systematic reviews, randomised controlled trials, non-controlled trials, quasi-experimental studies, cohort studies, case-control studies, outbreak reports, cross-sectional studies and surveillance reports.

- Studies providing data on any of the targeted pathogens: cephalosporin-resistant or carbapenem-resistant Enterobacteriaceae, carbapenem-resistant Pseudomonas aeruginosa, carbapenem-resistant Acinetobacter spp., vancomycin-resistant Enterococcus spp., methicillin-resistant Staphylococcus aureus.

- Studies providing information on any of the targeted infections: hospital-acquired pneumonia, ventilator-associated pneumonia, complicated intra-abdominal infections, complicated urinary tract infections or bloodstream infections.

- For data on the burden of infections: studies providing estimates or data on the frequency of targeted infections caused by MDR0s, including at least one of the following: prevalence, incidence rate or cumulative incidence, incidence density, percentage of resistant isolates.

- For data on outcomes of infections: studies providing data on outcomes of targeted infections caused by MDROs, including at least one of the following: mortality rates, length of stay.

- Time period and geographical scope: January 2005 through December 2018, European countries according to the ESCMID (European Society of Clinical Microbiology and Infectious Diseases) definition of geographical regions (https://www.escmid.org/fileadmin/src/media/PDFs/5Profession_Career/Parity_Commission/ ESCMID_Definition_of_Geographic_Regions_140703.pdf).

No age or language restrictions.

have been developed for this study using a combination of keywords (listed in box 2).

\section{Data management, selection process and data collection process}

A two-step selection procedure will be followed for the selection of studies. A single reviewer will screen the titles and abstracts of studies retrieved during the search strategy to discard ineligible studies (eg, case reports, case series, basic science studies). The full-text results of potentially eligible documents will be uploaded to Zotero software; duplicates will be removed. Two reviewers will assess these for relevance against the predefined selection criteria. The reference list of identified articles will be searched for potential additional studies.

The data will be extracted independently by two reviewers using a standardised electronic data extraction form, to be previously pilot tested on a representative sample. Disagreements will be resolved by a third reviewer. Data extraction from a $10 \%$ random sample of included articles will be checked by a third reviewer.

\section{Data items: primary end points and other variables}

The main end points to collect will be: (1) For burden of infections, incidence rate or density of infections (defined as the number of new cases of infection per 100 hospitalised patients and/or 1000 patient-days occurring in a specific time period, respectively) and prevalence

\section{Box 2 Keyword combinations for search strategy.}

Pathogens and resistance terms

- Target Gram negatives: 'multidrug-resistant' or 'MDR' or 'extensively-drug resistant' or 'XDR' or 'cephalosporin-resistance' or 'extended-spectrum beta-lactamase' or 'ESBL' or 'CTX-M' or 'SHV' or 'TEM' or 'AmpC' or 'imipenem-resistant' or 'meropenemresistant' or ertapenem-resistant' or 'carbapenemase' or 'KPC' or 'metallo-beta-lactamase' or 'NDM' or 'VIM' or 'IMP' or 'oxacillinase' or 'OXA-48' or 'OXA-48-like' or 'CRE' or 'CPE' or 'porin Ioss' AND 'Enterobacteria' or 'Enterobacteriaceae' or 'Klebsiella' or 'coli' or 'Proteus' or 'Morganella' or 'Citrobacter' or 'Serratia' or 'Enterobacter' or 'Pseudomonas' or 'Acinetobacter'

- Target Gram positives: 'multidrug-resistant' or 'MDR' or 'extensively drug-resistant' or 'XDR' or 'methicillin-resistant' or 'meticillinresistant' or 'oxacillin-resistant' or 'vancomycin-resistant' or 'MRSA' or 'VRE' AND 'Staphylococcus aureus' or 'Enterococcus' or 'enterococci'

\section{AND infection type terms}

- 'bacteremia' or 'bloodstream infection'

- 'healthcare-associated pneumonia' or 'ventilator-associated pneumonia' or 'hospital-acquired pneumonia' or 'pneumonia' or 'respiratory infections' or 'cystic fibrosis' or 'bronchiectasis'

- 'urinary tract infections' or 'UTI' or 'cystitis' or 'pyelonephritis' or 'catheter-associated urinary tract infections'

- 'intra-abdominal infection' or 'peritonitis' or 'cholangitis' or 'cholecystitis' or 'appendicitis' or 'intra-abdominal abscess' or 'IAl'

- 'SSSI' or 'skin and skin structures infections' or 'surgical site infections' or 'cellulitis' or 'skin and soft tissue infections'

\section{AND burden OR outcome}

- For burden of infections: 'prevalence' or 'incidence' or 'rate' or 'percentage of resistance' or 'proportion of resistance'

- For outcomes of infections: 'length of stay' or 'mortality' or 'death rate' or 'fatality' or 'survival rate' or 'death' or 'dead' or 'died'

(defined as the proportion of existing cases of infection per 100 patients admitted at a particular time point). (2) For clinical outcomes, crude and infection-attributable mortality, and length of hospital stay (overall and in intensive care units). Mortality will be collected as defined in each study, but the primary intention is to analyse in-hospital, 14-day, 30-day and 90-day mortalities. Also, length of stay will be collected as defined in the studies. Data on the proportion of resistant pathogens causing the infections will also be collected if available. We will calculate the end points even if not directly provided in the articles, provided the numerators and denominators are available. Other data to be collected are shown in table 1; for these, we will rely on the definitions used in the studies but will provide descriptive information about the definitions.

\section{Quality assessment}

The quality of the data retrieved will be evaluated by two reviewers using the following criteria: (1) The risk of selection bias (low, medium, high) for measuring burden of disease, in terms of ability to detect cases and representativeness of specific populations. (2) The risk of information bias (low, medium, high) based on definition of case, infection type and microbiological assessment. (3) The quality of randomised control trials will be measured 
Table 1 Variables to be collected from included studies

\begin{tabular}{|c|c|}
\hline Core element & Variable \\
\hline Study, site, time & First author, title, country, year(s) \\
\hline Scope $^{*}$ & One hospital, multicentre, region, country \\
\hline Population* & $\begin{array}{l}\text { Age: all/children/adults } \\
\text { Ward: all hospital ward, specific wards (ICU, etc) } \\
\text { Specific underlying conditions: neutropenia, dialysis, and so on }\end{array}$ \\
\hline Microorganism(s) ${ }^{*}$ & Genre and species, phenotypical resistance, mechanism(s) of resistance (if available) \\
\hline Epidemiological situation* & Outbreak, endemic situation \\
\hline End point variablest & $\begin{array}{l}\text { Burden: Prevalence, cumulative incidence, incidence density, proportion of resistant isolates } \\
\text { Clinical outcomes: all-cause mortality, infection-related mortality, length of hospital stay }\end{array}$ \\
\hline Mortality definitions* & In-hospital, fix-day \\
\hline
\end{tabular}

${ }^{*}$ As defined in the articles. †Definitions in the text.

. BSI, bloodstream infection; clAI, complicated intra-abdominal infection; cSSSI, complicated skin and skin structure infection; cUTI, complicated urinary tract infection; HAP, hospital-acquired pneumonia; ICU, intensive care unit; VAP, ventilator-associated pneumonia.

using the Effective Practice and Organisation of Care Scale, and the quality of non-randomised controlled trials with the Newcastle-Ottawa Scale.

\section{Data synthesis and analysis}

The collected data will be summarised in tabular form, indicating the core characteristics of surveillance systems and studies. For each pathogen and type of infection, estimates of rates and outcomes will be provided; stratification by geographical area, time period, population, epidemiological situation will be performed if possible. Pooled estimates of rates and outcomes with 95\% CIs will be provided; meta-analyses will be performed if possible with R statistical software (V.3.0.2; R Foundation for Statistical Computing, Vienna, Austria). The $I^{2}$ statistic will be used to measure the heterogeneity of estimates between studies, but because heterogeneity is expected, random-effects models will be used. Correlations between rates and outcomes in randomised controlled trials and epidemiological studies, and data on proportions and incidence rates will also be analysed. In addition, a description of definitions for the key variables used in the different studies will be provided.

\section{Patient and public involvement risk of bias}

Patients or public were not involved in the systematic review design.

\section{DISCUSSION}

Despite the epidemiological and clinical importance of infections caused by MDROs, comprehensive data about their burdens and outcomes are largely missing. Most information seems to come from single-centre studies in the context of outbreaks, or from surveillance data lacking patient-derived denominators.
Assessing the burden of the specific types of infection caused by these organisms is critically important in order to identify priorities for infection control activities and research. Understanding the geographical and population-type variability of the rates is also key for the design of clinical studies to evaluate the efficacy and safety of new or old antimicrobial drugs. Several recent studies have estimated the burden of infection and mortality caused by multidrug-resistant pathogens. ${ }^{712}$ Nevertheless, the estimates are based on limited and not necessarily reliable data, as has been underlined. ${ }^{1314}$ The heterogeneity of populations and the influence of multiple highly interconnected variables (infection types, comorbidities, severity of illness, appropriateness of therapy, etc), as well as study design and analysis methods mean that the evaluation of mortality attributable to resistance is highly variable. ${ }^{8-11}$ Source of data for rates of infections due to MDRO may be difficult to find. Data in the scientific literature may be limited because of publication bias, favouring reporting of outbreaks or data from university hospitals; therefore, we will also search the publicly available data from surveillance systems previously identified by the SUSPIRE project. Finally, as far as we know, there are no systematic reviews linking incidence rates with outcome of infection.

We expect some difficulties in our study and acknowledge some limitations. The data for some pathogens and infections may be scarce and sometimes based on special epidemiological situations; we would try to stratify the data according to the epidemiological situations but this may be difficult. The studies and surveillance systems may also be heterogeneous in the population studies, designs and quality. However, identifying gaps in knowledge will also be important for future studies. Definitions for outcomes, and methods for assessing them may be heterogeneous. 
We will also provide descriptive data about this which may be useful for future studies.

In summary, the aim of the ABOUT-MDRO project is to provide pooled estimates of target MDRO burdens and outcomes that are as specific as possible for different settings and populations as a tool to help policy makers and researchers establish priorities and design better studies for use in the fight against antimicrobial resistance. The study will also summarise grey literature data in order to assess the clinical burden of MDRO infections at the European level. These results will also be informative for the efficient design of randomised clinical trials.

\section{Author affiliations}

${ }^{1}$ Unidad Clínica de Enfermedades Infecciosas, Microbiología y Medicina Preventiva, Hospital Universitario Virgen Macarena / Departamento de Medicina, Universidad de Sevilla / Instituto de Biomedicina de Sevilla (IBiS), Sevilla, Spain

${ }^{2}$ Divisione di Malattie Infettive, Dipartimento di Diagnostica e Sanità Pubblica, Ospedale Policlinico Borgo Roma, Verona, Italy

Contributors JR-B and ET conceived the study. JR-B led the development of the protocol, provided supervision and mentorship to BA-B who wrote the first draft, and developed part of the search strategy, and coordinated and integrated ideas and comments from ZRP-B, NM and VP. The other coauthors MDP, SC, ER, $\mathrm{MC}$ developed the clinical outcomes part of the study protocol. All the authors contributed to the selection of variables and the rest of the protocol sections.

Funding The ABOUT-MDRO Study is part of the COMBACTE-MAGNET project (Combatting Bacterial Resistance in Europe - Molecules against Gram-negative Infections), Innovative Medicines Initiative (IMI), European Union's Seventh Framework Programme (FP7/2007-2013) and EFPIA companies' in-kind contribution, Grant agreement 115737. ZRP-B and JR-B received funding for research from Plan Nacional de I+D+i 2013-2016 and the Instituto de Salud Carlos III, Subdirección General de Redes y Centros de Investigación Cooperativa, Ministerio de Economía, Industria y Competitividad, Spanish Network for Research in Infectious Diseases (REIPI RD16/0016/0001), co-financed by the European Development Regional Fund 'A way to achieve Europe', Operative Programme Intelligent Growth 2014-2020.

Competing interests ZRP-B received honoraria for educational talks by Gilead. $\mathrm{JR}-\mathrm{B}$ received honoraria for accredited educational activities funded by Merck through unrestricted grants. All other authors declare that they have no conflicts of interest.

Patient and public involvement Patients and/or the public were not involved in the design, or conduct, or reporting, or dissemination plans of this research.

Patient consent for publication Not required.

Provenance and peer review Not commissioned; externally peer reviewed.

Data availability statement There are no data in this work.

Open access This is an open access article distributed in accordance with the Creative Commons Attribution Non Commercial (CC BY-NC 4.0) license, which permits others to distribute, remix, adapt, build upon this work non-commercially, and license their derivative works on different terms, provided the original work is properly cited, appropriate credit is given, any changes made indicated, and the use is non-commercial. See: http://creativecommons.org/licenses/by-nc/4.0/.

\section{ORCID iDs}

Zaira R Palacios-Baena http://orcid.org/0000-0002-1713-6807

Sheila Chiesi http://orcid.org/0000-0002-0108-0722

\section{REFERENCES}

1 European Commission. Action plan against the rising threats from antimicrobial resistance. In: Communication from the Commission to the European Parliament and the Council. 15. Brussels, 2011.

2 The bacterial challenge: time to react. ECDC/EMEA joint technical report. EMEA Doc. Ref. EMEA/576176/2009. Stockholm, September 2009.ISBN 978-92-9193-193-4. Available: https://ecdc.europa.eu/ sites/portal/files/media/en/publications/Publications/0909_TER_The_ Bacterial_Challenge_Time_to_React.pdf [Accessed March 2019].

3 Tacconelli E, Carrara E, Savoldi A, et al. Discovery, research, and development of new antibiotics: the who priority list of antibiotic-resistant bacteria and tuberculosis. Lancet Infect Dis 2018;18:318-27.

4 Tacconelli E, Sifakis F, Harbarth S, et al. Surveillance for control of antimicrobial resistance. Lancet Infect Dis 2018;18:e99-106.

5 Núñez-Núñez M, Navarro MD, Palomo V, et al. The methodology of surveillance for antimicrobial resistance and healthcare-associated infections in Europe (SUSPIRE): a systematic review of publicly available information. Clin Microbiol Infect 2018;24:105-9.

6 Babu Rajendran N, Gladstone BP, Rodríguez-Baño J, et al. Epidemiology and control measures of outBreaks due to antibiotic-resistant orGanisms in Europe (embargo): a systematic review protocol 6A Beryl P, Vilken T, Bernhard a, Tacconelli E. epidemiological differences in controlling the spreading of carbapenem-resistant bacterial strains in hospitalized patients. ECCMID 2016 Amsterdam, O386 (oral presentation).. BMJ Open 2017;7:e013634.

7 O'Neill J. Antimicrobial resistance: tackling a crisis for the health and wealth of nations. Available: https://amr review.org/sites/default/files/ AMR\% 20Review \%20Paper\%20\%20Tackling\%20a\%20crisis\% 20for\%20the\%20health\%20and\%20wealth\%20of\%20nations_1.pdf

8 Zeng X, Zhang Y, Kwong JSW, et al. The methodological quality assessment tools for preclinical and clinical studies, systematic review and meta-analysis, and clinical practice guideline: a systematic review. J Evid Based Med 2015;8:2-10.

9 Vardakas KZ, Rafailidis PI, Konstantelias AA, et al. Predictors of mortality in patients with infections due to multi-drug resistant gram negative bacteria: the study, the patient, the bug or the drug? $J$ Infect 2013;66:401-14.

10 Naylor NR, Atun R, Zhu N, et al. Estimating the burden of antimicrobial resistance: a systematic literature review. Antimicrob Resist Infect Control 2018;7:58.

11 Evans SR, Harris AD. Methods and issues in studies of Cre. Virulence 2017;8:453-9.

12 Cassini A, Högberg LD, Plachouras D, et al. Attributable deaths and disability-adjusted life-years caused by infections with antibioticresistant bacteria in the EU and the European economic area in 2015: a population-level modelling analysis. Lancet Infect Dis 2019;19:56-66.

13 de Kraker MEA, Stewardson AJ, Harbarth S. Will 10 million people die a year due to antimicrobial resistance by 2050? PLoS Med 2016;13:e1002184.

14 Tacconelli E, Pezzani MD. Public health burden of antimicrobial resistance in Europe. Lancet Infect Dis 2019;19:4-6. 